\title{
Distinguishing a set of full product bases needs only projective measurements and classical communication
}

\author{
Ping-Xing Chen ${ }^{1,2 *}$ and Cheng-Zu Li ${ }^{2}$ \\ 1. Laboratory of Quantum Communication and Quantum Computation, \\ University of Science and Technology of \\ China, Hefei, 230026, P. R. China. \\ 2. Department of Applied Physics, National University of \\ Defense Technology, \\ Changsha, 410073, \\ P. R. China.
}

(October 30, 2018)

\begin{abstract}
Nonlocality without entanglement is an interesting field. A manifestation of quantum nonlocality without entanglement is the local indistinguishability of a set of orthogonal product states. In this paper we analyze the character of operators to distinguish a set of full product bases in a multi-partite system, and show that distinguishing perfectly a set of full product bases needs only local projective measurements and classical communication, and these measurements cannot damage each product basis. Employing these conclusions one can discuss local distinguishability of full product bases easily. Finally we discuss the generalization of these results to the locally distinguishability of a set of incomplete product bases.
\end{abstract}

PACS number(s): 89.70.+c, 03.65.ud

Typeset using REVTEX

*E-mail: pxchen@nudt.edu.cn 
An important manifestation of quantum nonlocality is entanglement [1]. The entangled states can be used for novel forms of information processing, such as quantum cryptography $[2,3]$, quantum teleportation [4], and fast quantum computation [5]. However, there also exists nonlocality in inentangled states [6,7], even in a state of a particle [3]. This is known as nonlocality without entanglement. The protocols of single photon cryptography [3] are examples which uses the nonlocality without entanglement. The nonlocality without entanglement may be an important field just as the entanglement. Closely related to the nonlocality without entanglement is the local distinguishability of a set of inentangled states $[6,7]$.

Alice, Bob and Charles et al share a quantum system, in one of a known set of possible orthogonal states. They do not, however, know which state they have. These states are locally distinguishable if there are some sequence of local operations and classical communication (LOCC) by which Alice, Bob and Charles et al can always determine which state they own. There are many interesting works on the local distinguishability of orthogonal states [6-15]. These works improve our understanding on nonlocality. The discussion on the local distinguishability of orthogonal product states (OPSs) may enlarge our acknowledge of nonlocality without entanglement. Bennett et al first [6] showed that there are 9 OPSs in a $3 \otimes 3$ system which are indistinguishable by LOCC. Walgate et al [7] provide a more simple proof of indistinguishability of the Bennett's 9 OPSs. However few papers discussed the local distinguishability of more general OPSs in a multi-partite system.

This paper will focus on the local distinguishability of a set of complete OPSs $\left\{\left|\Psi_{k}\right\rangle\right\}$ in a multi-partite system. We will show that a set of full OPSs are LOCC perfectly distinguishable if and only if these OPSs are distinguishable by projective measurements and classical communication, and these measurements cannot damage each state $\left|\Psi_{k}\right\rangle$. Using this result we can prove easily that the Bennett's 9 OPSs [6] are indistinguishable by LOCC, and can provide many new sets of locally indistinguishable OPSs in multi-partite systems. Finally we discuss the generalization of these results to the LOCC distinguishability of incomplete product bases.

Alice, Bob and Charles et al share a quantum system which may be in one of the possible states $\left\{\left|\psi_{k}\right\rangle, k=1, \cdots, M\right\}$. Any protocol to distinguish these possible states can be conceived as successive rounds of measurements and communication by Alice, Bob and Charles et al. After $N(N \geqslant 1)$ rounds of measurements and communication, there are many possible outcomes which correspond to many measurement operators $\left\{A_{i N} \otimes B_{i N} \otimes\right.$ $\left.C_{i N} \otimes \cdots\right\}$ acting on the Alice, Bob and Charles's Hilbert space. Each of these operators is a product of the positive operators and unitary maps corresponding to Alice's, Bob's and Charles's measurement and rotations, and represents the effect of the $\mathrm{N}$ measurements and communication. If the outcome $i N$ occurs, the given state $\left|\psi_{k}\right\rangle$ becomes $[7,16]$ : 


$$
\left|\psi_{k}\right\rangle \rightarrow A_{i N} \otimes B_{i N} \otimes C_{i N} \otimes \cdots\left|\psi_{k}\right\rangle
$$

Operator $A_{i N}, B_{i N}, C_{i N}$ can be expressed as (see Appendix in this paper, or Ref. [17]):

$$
\begin{gathered}
A_{i N}=c_{1}^{i N}\left|\phi_{1}^{i N}\right\rangle\left\langle\phi_{1}^{i N}\left|+\cdots+c_{n_{a}^{i N}}^{i N}\right| \phi_{n_{a}^{i N}}^{i N}\right\rangle\left\langle\phi_{n_{a}^{i N}}^{i N}\right| ; \\
B_{i N}=d_{1}^{i N}\left|\xi_{1}^{i N}\right\rangle\left\langle\xi_{1}^{i N}\left|+\cdots+d_{n_{b}^{i N}}^{i N}\right| \xi_{n_{b}^{i N}}^{i N}\right\rangle\left\langle\xi_{n_{b}^{i N}}^{i N}\right| ; \\
C_{i N}=e_{1}^{i N}\left|\eta_{1}^{i N}\right\rangle\left\langle\eta_{1}^{i N}\left|+\cdots+e_{n_{c}^{i N}}^{i N}\right| \eta_{n_{c}^{i N}}^{i N}\right\rangle\left\langle\eta_{n_{c}^{i N}}^{i N}\right| ;
\end{gathered}
$$

where $\left\{\left|\phi_{j}^{i N}\right\rangle, j=1, \cdots, n_{a}^{i N}\right\},\left\{\left|\phi_{j}^{i N}\right\rangle, j=1, \cdots, n_{a}^{i N}\right\}$ are Alice's two set of orthogonal vectors; $\left\{\left|\xi_{l}^{\prime N}\right\rangle, l=1, \cdots, n_{b}^{i N}\right\},\left\{\left|\xi_{l}^{i N}\right\rangle, l=1, \cdots, n_{b}^{i N}\right\}$ are Bob's two set of orthogonal vectors; $\left\{\left|\eta_{p}^{\prime i N}\right\rangle, p=1, \cdots, n_{c}^{i N}\right\},\left\{\left|\eta_{p}^{i N}\right\rangle, p=1, \cdots, n_{c}^{i N}\right\}$ are Charles's two set of orthogonal vectors. $0 \leq c_{j}^{i N} \leq 1, j=1, \cdots, n_{a}^{i N} ; 0 \leq d_{l}^{i N} \leq 1, l=1, \cdots, n_{b}^{i N} ; 0 \leq e_{p}^{i N} \leq$ $1, p=1, \cdots, n_{c}^{i N}$. The operator $A_{i N}$ in (2) can be carried out by following three operators: 1) a projective operator which projects out $\left.\left|\phi_{j}^{i N}\right\rangle, j=1, \cdots, n_{a}^{i N} ; 2\right)$ a local filter operator which changes the relative weights of the components $\left.\left|\phi_{j}^{i N}\right\rangle, j=1, \cdots, n_{a}^{i N} ; 3\right)$ a local unitary operator which transfer the Alice's vectors from $\left\{\left|\phi_{j}^{i N}\right\rangle, j=1, \cdots, n_{a}^{i N}\right\}$ to $\left\{\left|\phi_{j}^{i N}\right\rangle, j=1, \cdots, n_{a}^{i N}\right\}$, and similarly for $B_{i N}$ and $C_{i N}$. Obviously, the effect of the local unitary operator 3 ) is to change the bases of the subspace projectived out by the projective operator 1), and does not affect the distinguishability of the possible states $\left\{\left|\psi_{k}\right\rangle, k=1, \cdots, M\right\}$. So $A_{i N}$ in (2) can be replaced by

$$
A_{i N}=c_{1}^{i N}\left|\phi_{1}^{i N}\right\rangle\left\langle\phi_{1}^{i N}\left|+\cdots+c_{n_{a}^{i N}}^{i N}\right| \phi_{n_{a}^{i N}}^{i N}\right\rangle\left\langle\phi_{n_{a}^{i N}}^{i N}\right|,
$$

and similarly for $B_{i N}$ and $C_{i N}$.

Definition 1: For each operator $A_{i N} \otimes B_{i N} \otimes C_{i N} \otimes \cdots$ in (1), if states $\left\{A_{i N} \otimes B_{i N} \otimes C_{i N} \otimes \cdots\left|\psi_{k}\right\rangle, k=1, \cdots, M\right\}$ are LOCC distinguishable, we say that operator $A_{i N} \otimes B_{i N} \otimes C_{i N} \otimes \cdots$ is effective to distinguish the states $\left\{\left|\psi_{k}\right\rangle\right\}$.

Theorem $1\left\{\left|\Psi_{k}\right\rangle, k=1, \cdots, M\right\}$ is a set of complete orthogonal product states in a multi-partite system. The states $\left\{\Psi_{k}\right\}$ are LOCC perfectly distinguishable if and only if the states are distinguishable by projective measurements and classical communication, and these measurements cannot damage each state $\left|\Psi_{k}\right\rangle$.

Proof: We first prove theorem 1 for the cases of bi-partite systems. The sufficiency is obvious. We need only prove the necessity. Suppose that Alice and Bob share a $n \otimes m$ system which has $n m$ possible OPSs $\left\{\left|\Psi_{k}\right\rangle=\left|v_{k}\right\rangle_{A}\left|y_{k}\right\rangle_{B}, k=1, \cdots, n m\right\}$, where $\left|v_{k}\right\rangle_{A},\left|y_{k}\right\rangle_{B}$ is a state of Alice's and Bob's, respectively. If the set of states $\left\{\Psi_{k}\right\}$ is perfectly distinguishable by LOCC, there must be a complete set of final operators $\left\{A_{i f} \otimes B_{i f}\right\}$ representing 
the effect of all measurements and communication, such that if every outcome if occurs Alice and Bob know with certainty that they were given the state $\left|\Psi_{i}\right\rangle \in\left\{\left|\Psi_{k}\right\rangle\right\}$. This means that:

$$
\begin{aligned}
& A_{i f} \otimes B_{i f}\left|\Psi_{i}\right\rangle \neq 0 \\
& A_{i f} \otimes B_{i f}\left|\Psi_{j}\right\rangle=0, j \neq i .
\end{aligned}
$$

Operators $A_{i f}, B_{i f}$ have similar forms as $A_{i N}, B_{i N}$ in (3) but for $N \rightarrow f$. We note that $A_{i f} \otimes B_{i f}$ can "indicates" $\left|\Psi_{i}\right\rangle$ and only $\left|\Psi_{i}\right\rangle$ [15]. Since the number of the operators satisfying the (4) is bigger than 1 , a state $\left|\Psi_{i}\right\rangle$ can be "indicated" by more than a operator, in general. By the general expressions $A_{i f}, B_{i f}$ as in (3) if operator $A_{i f} \otimes B_{i f}$ can "indicates" $\left|\Psi_{i}\right\rangle$ and only $\left|\Psi_{i}\right\rangle$, i.e., (4) holds, the state $\left|\Psi_{i}\right\rangle$ should contain all or part of orthogonal product vectors in the following:

$$
\left|\phi_{1}^{i f}\right\rangle\left|\xi_{1}^{i f}\right\rangle, \cdots,\left|\phi_{1}^{i f}\right\rangle\left|\xi_{n_{b}^{i f}}^{i f}\right\rangle, \cdots,\left|\phi_{n_{a}^{i f}}^{i f}\right\rangle\left|\xi_{1}^{i f}\right\rangle, \cdots,\left|\phi_{n_{a}^{i f}}^{i f}\right\rangle\left|\xi_{n_{b}^{i f}}^{i f}\right\rangle
$$

and $\left|\Psi_{j}\right\rangle(j \neq i)$ do not contain any product vectors in $(5)$, i.e., each product vectors in $(5)$ is orthogonal to $\left|\Psi_{j}\right\rangle(j \neq i)$. Since for a $n \otimes m$ system the vector which is orthogonal to $n m-1$ orthogonal states $\left|\Psi_{j}\right\rangle(j \neq i)$ is alone, if operator $A_{i f} \otimes B_{i f}$ "indicates" $\left|\Psi_{i}\right\rangle$, then $\left|\Psi_{i}\right\rangle$ contains only one product vector in (5). Namely, $\left|\Psi_{i}\right\rangle$ should be one of the product vectors in (5). So all operators $A_{i f} \otimes B_{i f}$ "indicating" $\left|\Psi_{i}\right\rangle$ and only $\left|\Psi_{i}\right\rangle$ should project out a intact state $\left|\Psi_{i}\right\rangle$, but not a component of $\left|\Psi_{i}\right\rangle$, and then all OPS in the set $\left\{\left|\Psi_{k}\right\rangle\right\}$ are eigenvectors of each operator in operators $\left\{A_{i f} \otimes B_{i f}\right\}$. For the same reason, we can prove that all OPSs in the set $\left\{\left|\Psi_{k}\right\rangle\right\}$ are eigenvectors of each operator $A_{i N} \otimes B_{i N}$ representing the effect of $\mathrm{N}$ round measurements and communication, $N=1,2, \cdots$. In fact, suppose that during the first measure to distinguish the states $\left\{\left|\Psi_{k}\right\rangle\right\}$ (suppose Alice do the first measure) if an effective operator $A_{i 1}$ does not project out an intact OPS, but a part of a OPS, then the two orthogonal parts of the OPS are orthogonal to $n m-1$ orthogonal states. This is impossible. On the other hand, after Alice and Bob finished the first measure and get a outcome, the whole space collapses into a subspace and the OPSs in this subspace form a set of complete bases of the subspace. So the sequent measures have same property as the first measure.

Let's now prove that the set of final operators $\left\{A_{i f} \otimes B_{i f}\right\}$ can be carried out by projective measurements and classical communication. To achieve this, we consider the Alice's first measurement described by $\left\{A_{i 1}\right\}$

$$
A_{i 1}=c_{1}^{i 1}\left|\phi_{1}^{i 1}\right\rangle_{A}\left\langle\phi_{1}^{i 1}\left|+\cdots+c_{n_{a}^{i 1}}^{i 1}\right| \phi_{n_{a}^{i 1}}^{i 1}\right\rangle_{A}\left\langle\phi_{n_{a}^{i 1}}^{i 1}\right|
$$

Operator $A_{i 1}$ project out a subspace of Alice spanned by Alice's bases $\left|\phi_{1}^{i 1}\right\rangle_{A}, \cdots,\left|\phi_{n_{a}^{i 1}}^{i 1}\right\rangle_{A}$. This subspace should contain some intact Alice's vectors of the OPSs (since $A_{i 1}$ should 
project out some intact OPSs). Since all OPSs $\left\{\left|\Psi_{k}\right\rangle\right\}$ are eigenvectors of the operator $A_{i 1}$, if $\left\{A_{i 1}\right\}$ is effective to distinguish the states $\left\{\left|\Psi_{k}\right\rangle\right\}$ then so does operators $\left\{A_{i 1}^{\prime}\right\}$ :

$$
A_{i 1}^{\prime}=\left|\phi_{1}^{i 1}\right\rangle\left\langle\phi_{1}^{i 1}|+\cdots+| \phi_{n_{a}^{i 1}}^{i 1}\right\rangle\left\langle\phi_{n_{a}^{i 1}}^{i 1}\right| .
$$

Operator $A_{i 1}^{\prime}$ projects out a same subspace $\varkappa_{i}$ as $A_{i 1}$ does, and all OPSs $\left\{\left|\Psi_{i}\right\rangle\right\}$ are the eigenvectors of the operator $A_{i 1}^{\prime}$. If operators $\left\{A_{i 1}^{\prime}\right\}$ is not a set of projective operators, we can find a set of projective operators $\left\{A_{i 1}^{\prime \prime}\right\}$ by following protocol such that if $\left\{A_{i 1}^{\prime}\right\}$ is effective to distinguish the states $\left\{\left|\Psi_{k}\right\rangle\right\}$ then so does operators $\left\{A_{i 1}^{\prime \prime}\right\}$. We first choose two operators $A_{11}^{\prime}, A_{21}^{\prime}$ described by $A_{i 1}^{\prime}(i=1,2)$ in $(7)$. Operators $A_{11}^{\prime}, A_{21}^{\prime}$ projects out subspace $\varkappa_{1}, \varkappa_{2}$, respectively. Both $\varkappa_{1}$ and $\varkappa_{2}$ should contains intact Alice's vectors of some OPSs $\left|\Psi_{k}\right\rangle$. Suppose $\varkappa_{1}$ and $\varkappa_{2}$ contains the Alice's vectors of $\left|\Psi_{k 1}\right\rangle s$ and $\left|\Psi_{k 2}\right\rangle s$, respectively, $\left|\Psi_{k 1}\right\rangle s,\left|\Psi_{k 2}\right\rangle s \in\left\{\left|\Psi_{k}\right\rangle\right\}$. The Alice's vectors of the OPSs belonging to $\left|\Psi_{k 2}\right\rangle s$ but not to $\left|\Psi_{k 1}\right\rangle s$ form a subspace $\varkappa$ of $\varkappa_{2}$. Obviously, $\varkappa$ is orthogonal to $\varkappa_{1}$. We note operator $A_{21}^{\prime \prime}$ projects out subspace $\varkappa$ and only $\varkappa$ ( In fact, $\varkappa_{2}=\varkappa \cup\left(\varkappa_{2} \cap \varkappa_{1}\right.$ ), and $\varkappa$ is orthogonal to $\left(\varkappa_{2} \cap \varkappa_{1}\right)$. Operator $A_{21}^{\prime}$ projects out subspace $\varkappa_{2}$. The effect of operator $A_{21}^{\prime \prime}$ is to discard some bases of $\varkappa_{2}$, and to project out subspace $\varkappa$ ). If we replace $A_{21}^{\prime}$ by $A_{21}^{\prime \prime}$, then $A_{11}^{\prime}$ and $A_{21}^{\prime \prime}$ project out some OPSs as same as $A_{11}^{\prime}$ and $A_{21}^{\prime}$ do, and these OPSs are distinguishable by LOCC if $A_{11}^{\prime}$ and $A_{21}^{\prime}$ are effective to distinguish the states $\left\{\left|\Psi_{k}\right\rangle\right\}$. Similarly, operator $A_{31}^{\prime}$ projects out subspace $\varkappa_{3}$. we can discard the bases of $\varkappa_{3}$ which are vectors of subspace $\varkappa_{1}$ or $\varkappa_{2}$. The left bases of $\varkappa_{3}$ span a subspace projected out by operator $A_{31}^{\prime \prime}$. By a sequence of similar operations we find always a set of orthogonal projective operators $\left\{A_{11}^{\prime}, A_{21}^{\prime \prime}, A_{31}^{\prime \prime}, \cdots\right\}$ such that if operators $\left\{A_{i 1}^{\prime}\right\}$ are effective to distinguish the OPSs, so do operators $\left\{A_{11}^{\prime}, A_{21}^{\prime \prime}, A_{31}^{\prime \prime}, \cdots\right\}$. Obviously $A_{11}^{\prime+} A_{11}^{\prime}+A_{21}^{\prime \prime+} A_{21}^{\prime \prime}+A_{31}^{\prime \prime+} A_{31}^{\prime \prime}+\cdots=I$. So Alice's first measure can be carried out by a set projective operators $\left\{A_{11}^{\prime}, A_{21}^{\prime \prime}, A_{31}^{\prime \prime}, \cdots\right\}$, similarly for Bob's first measure. On the other hand, after Alice and Bob finished the first measure and get a outcome, the whole space collapses into a subspace and the OPSs in this subspace form a set of complete bases of the subspace. So the sequent measures have same property as the first measure. Thus the set of operators $\left\{A_{i f} \otimes B_{i f}\right\}$ can be carried out by projective measurements and classical communication. The whole proof is completely fit to the cases of multi-partite systems. This ends the proof.

Definition 2: If two states $\left|\Phi_{1}\right\rangle$ and $\left|\Phi_{2}\right\rangle$ satisfying $\left\langle\Phi_{1} \mid \Phi_{2}\right\rangle \neq 0$, we say $\left|\Phi_{1}\right\rangle$ and $\left|\Phi_{1}\right\rangle$ are relative. This is noted as $\left|\Phi_{1}\right\rangle \longleftrightarrow\left|\Phi_{2}\right\rangle$; if $\left|\Phi_{1}\right\rangle \longleftrightarrow\left|\Phi_{2}\right\rangle \longleftrightarrow \cdots \longleftrightarrow$ $\left|\Phi_{N}\right\rangle$ (i.e., $\left.\left\langle\Phi_{1} \mid \Phi_{2}\right\rangle \neq 0 ;\left\langle\Phi_{2} \mid \Phi_{3}\right\rangle \neq 0 ; \cdots ;\left\langle\Phi_{N-1} \mid \Phi_{N}\right\rangle \neq 0\right)$, we say $\left|\Phi_{1}\right\rangle,\left|\Phi_{2}\right\rangle, \cdots,\left|\Phi_{N}\right\rangle$ are relative.

Theorem 2: A set of states $\left\{\left|v_{i}\right\rangle_{A}\left|y_{i}\right\rangle_{B}, i=1, \cdots, n m\right\}$ is $n m$ OPSs in a $n \otimes m$ system. If for every given state $\left|v_{i}\right\rangle_{A}\left|y_{i}\right\rangle_{B}$, there are $n-1$ states $\left|v_{j}^{\prime}\right\rangle_{A}\left|y_{j}^{\prime}\right\rangle_{B} \in\left\{\left|v_{i}\right\rangle_{A}\left|y_{i}\right\rangle_{B}, i=\right.$ $1, \cdots, n m\}, j=1, \cdots, n-1$ such that

$$
\left|v_{i}\right\rangle_{A} \longleftrightarrow\left|v_{1}^{\prime}\right\rangle_{A} \longleftrightarrow \cdots \longleftrightarrow\left|v_{n-1}^{\prime}\right\rangle_{A},
$$


and $\left|v_{i}\right\rangle_{A},\left|v_{1}^{\prime}\right\rangle_{A}, \cdots,\left|v_{n-1}^{\prime}\right\rangle$ are linearly independent; If for every given state $\left|v_{i}\right\rangle_{A}\left|y_{i}\right\rangle_{B}$, there are $m-1$ states $\left|v_{k}^{\prime}\right\rangle_{A}\left|y_{k}^{\prime}\right\rangle_{B} \in\left\{\left|v_{i}\right\rangle_{A}\left|y_{i}\right\rangle_{B}, i=1, \cdots, n m\right\}, k=1, \cdots, m-1$ such that

$$
\left|y_{i}\right\rangle_{B} \longleftrightarrow\left|y_{1}^{\prime}\right\rangle_{B} \longleftrightarrow \cdots \longleftrightarrow\left|y_{m-1}^{\prime}\right\rangle_{B}
$$

and $\left|y_{i}\right\rangle_{B},\left|y_{1}^{\prime}\right\rangle_{B}, \cdots,\left|y_{m-1}^{\prime}\right\rangle_{B}$ are linearly independent, then states $\left\{\left|v_{i}\right\rangle_{A}\left|y_{i}\right\rangle_{B}, i=\right.$ $1, \cdots, n m\}$ are not LOCC distinguishable.

Proof: Suppose Alice do the first measure (Alice goes first [7]). From theorem 1 it follows that all states $\left\{\left|v_{i}\right\rangle_{A}\left|y_{i}\right\rangle_{B}, i=1, \cdots, n m\right\}$ are eigenstates of Alice's first measure described as $A_{j}$ in (7). If $\left|v_{i}\right\rangle_{A}$ is a eigenstate of operator $A_{j}$ with non-zero eigenvalue, equation (8) implies that $\left|v_{1}^{\prime}\right\rangle_{A}$ should be also a eigenstate of operator $A_{j}$ with non-zero eigenvalue, and so does $\left|v_{j}^{\prime}\right\rangle_{A}, j=2, \cdots, n-1$. So the rank of operator $A_{j}$ is full. A full-rank-operator $A_{j}$ would project out all OPSs and can do nothing to distinguish states $\left\{\left|v_{i}\right\rangle_{A}\left|y_{i}\right\rangle_{B}, i=1, \cdots, n m\right\}$, and similarly for Bob's first measure. So the states $\left\{\left|v_{i}\right\rangle_{A}\left|y_{i}\right\rangle_{B}, i=1, \cdots, n m\right\}$ are not LOCC distinguishable. This ends the proof.

Theorem 2 above can be generalized into multi-partite cases, obviously. From the theorem 2 we can get many cases of indistinguishable states. There are three examples in the following:

Case 1 The 9 OPSs in a $3 \otimes 3$ system in the following are indistinguishable as shown in paper [6] of Bennett et al.

$$
\begin{aligned}
\left|\Psi_{1}\right\rangle & =|1\rangle_{A}|1\rangle_{B} ;\left|\Psi_{2,3}\right\rangle=|3\rangle_{A}|3 \pm 1\rangle_{B} \\
\left|\Psi_{4,5}\right\rangle & =|2\rangle_{A}|1 \pm 2\rangle_{B} ;\left|\Psi_{6,7}\right\rangle=|3 \pm 1\rangle_{A}|2\rangle_{B} \\
\left|\Psi_{8,9}\right\rangle & =|1 \pm 2\rangle_{A}|3\rangle_{B}
\end{aligned}
$$

Case 2 The following 16 OPSs in a $4 \otimes 4$ system are indistinguishable.

$$
\begin{aligned}
\left|\Psi_{1,2}\right\rangle & =|1\rangle_{A}|1 \pm 2\rangle_{B} ; & & \left|\Psi_{3,4}\right\rangle=|2\rangle_{A}|2 \pm 3\rangle_{B} \\
\left|\Psi_{5,6}\right\rangle & =|3\rangle_{A}|3 \pm 4\rangle_{B} ; & & \left|\Psi_{7,8}\right\rangle=|4\rangle\left|{ }_{A} 1 \pm 4\right\rangle_{B} \\
\left|\Psi_{9,10}\right\rangle & =|1 \pm 2\rangle_{A}|4\rangle_{B} ; & & \left|\Psi_{11,12}\right\rangle=|3 \pm 4\rangle_{A}|2\rangle_{B} \\
\left|\Psi_{13,14}\right\rangle & =|2 \pm 3\rangle_{A}|1\rangle_{B} ; & & \left|\Psi_{15,16}\right\rangle=|1 \pm 4\rangle_{A}|3\rangle_{B}
\end{aligned}
$$

Case 3 The following 64 OPSs in a $4 \otimes 4 \otimes 4$ system are indistinguishable.

$$
\begin{aligned}
\left|\Psi_{i}\right\rangle & =\left|\Psi_{i}\right\rangle_{A B}|1\rangle_{C} ; & & \left|\Psi_{i+16}\right\rangle=\left|\Psi_{i}\right\rangle_{A B}|2\rangle_{C} \\
\left|\Psi_{i+32}\right\rangle & =\left|\Psi_{i}\right\rangle_{A B}|3\rangle_{C} ; & & \left|\Psi_{i+48}\right\rangle=\left|\Psi_{i}\right\rangle_{A B}|4\rangle_{C} \\
i & =1, \cdots, 16 & &
\end{aligned}
$$

Where $\left|\Psi_{i}\right\rangle$ is a state in case 2 . 
Employing the above theorem 2, we can prove the cases 1 and 2 easily. In case 3, Charles can do the first projective measurement by operators $|1\rangle\langle 1|| 2\rangle,\langle 2|| 3\rangle,\langle 3|| 4\rangle,\langle 4|$. But after Charles's first round measurement the states of Alice and Bob' part will collapse into some indistinguishable OPSs $\left|\Psi_{i}\right\rangle_{A B} s$. So the OPSs in case 3 is indistinguishable by LOCC.

Locally distinguishing a set of full OPSs needs only local projective measurements and classical communication. Can this conclusion be generalized into a set of incomplete OPSs? A set of following OPSs shows it is not always true. Nine OPSs [18]

$$
\begin{aligned}
& \left|\Psi_{1,2,3}\right\rangle=\left|\Psi_{1,2,3}\right\rangle_{A B}|x\rangle_{C} \\
& \left|\Psi_{4,5,6}\right\rangle=\left|\Psi_{4,5,6}\right\rangle_{A B}|y\rangle_{C} \\
& \left|\Psi_{7,8,9}\right\rangle=\left|\Psi_{7,8,9}\right\rangle_{A B}|z\rangle_{C}
\end{aligned}
$$

where $\left|\Psi_{i}\right\rangle(i=1, \cdots, 9)$ is a state in $(10),|x\rangle=|1\rangle ;|y\rangle=(|1\rangle+\sqrt{3}|2\rangle) / 2 ;|z\rangle=(|1\rangle-$ $\sqrt{3}|2\rangle) / 2$, can be distinguished by Charles doing the first measure described by operators $C_{i 1}(i=1,2,3)[18]$

$$
\begin{aligned}
& C_{11}=\sqrt{\frac{2}{3}}\left|x^{*}><x^{*}\right| ; \\
& C_{21}=\sqrt{\frac{2}{3}}\left|y^{*}><y^{*}\right| ; \\
& C_{31}=\sqrt{\frac{2}{3}}\left|z^{*}><z^{*}\right|,
\end{aligned}
$$

where $\left\langle x \mid x^{*}\right\rangle=\left\langle y \mid y^{*}\right\rangle=\left\langle z \mid z^{*}\right\rangle=0$, and $\sum_{i=1}^{3} C_{i 1}^{+} C_{i 1}=1$. After Charles get a outcome, 9 OPSs in (13) collapse into locally distinguishable 6 OPSs. However, states $\left|\Psi_{i}\right\rangle(i=1, \cdots, 9)$ in (13) cannot be distinguished by local projective measurements and classical communication.

In conclusion, we analyze the character of operators to distinguish a set of full OPSs in a multi-partite system, and show that to distinguish perfectly a set of full bases needs only local projective measurements and classical communication, and these measurements cannot damage each OPS. Employing these conclusions one can discuss local distinguishability of full product bases easily. An open question is that whether these conclusions can be generalized to the local distinguishability of states in a quantum system, the sum of Schmidt number of the states is equal to the dimensions of Hilbert space of the system. Another open question is that which classes of operators can be carried out only local projective measurements, since local projective measurements are easier to be achieved than generalized POV measurements. 


\section{ACKNOWLEDGMENTS}

We would like to thank J. Finkelstein for presenting us a set of special states in (13) and Guangcan Guo for his help to this work.

Appendix

Now we will prove that operator $A_{i N}, B_{i N}, C_{i N}$ can be expressed as the form in (2). To this end, note that we can always write an arbitrary operator $A_{i N}$ in the form [17]

$$
A_{i N}=\left|\beta_{1}^{i N}\right\rangle\left\langle\alpha_{1}^{i N}|+\cdots+| \beta_{n_{a}^{i N}}^{i N}\right\rangle\left\langle\alpha_{n_{a}^{i N}}^{i N}\right|,
$$

where $\left\{\left|\alpha_{1}^{i N}\right\rangle, \cdots,\left|\alpha_{n_{a}^{i N}}^{i N}\right\rangle\right\}$ is a set of Alice's orthogonal and normalized vectors; $\left\{\left|\beta_{1}^{i N}\right\rangle, \cdots,\left|\beta_{n_{a}^{i N}}^{i N}\right\rangle\right\}$ is a set of linearly independent (possibly unnormalized) Alice's vectors $[19]$. We take another set of orthogonal and normalized vectors $\left\{\left|\alpha_{1}^{i N}\right\rangle, \cdots,\left|\alpha_{n_{a}^{i N}}^{i N}\right\rangle\right\}$ satisfying

$$
\left[\left|\alpha^{i N}\right\rangle\right]=[u]\left[\left|\alpha^{i N}\right\rangle\right]
$$

where

$$
\left[\left|\alpha^{i N}\right\rangle\right]=\left[\begin{array}{c}
\left|\alpha_{1}^{i N}\right\rangle \\
\vdots \\
\left|\alpha_{n_{a}^{i N}}^{i N}\right\rangle
\end{array}\right]
$$

and similarly for $\left[\left|\alpha^{i N}\right\rangle\right],\left[\left|\beta^{i N}\right\rangle\right]$ and $\left[\left|\beta^{\prime i N}\right\rangle\right]$ below; $[u]$ is a unitary matrix. Eq. (16) can be expressed as explicitly

$$
\left|\alpha_{j}^{i N}\right\rangle=\sum_{k=1}^{n_{a}^{i N}} u_{j k}\left|\alpha_{k}^{i N}\right\rangle, j=1, \ldots, n_{a}^{i N},
$$

where $u_{j k}$ is an element of the unitary matrix $[u]$.

Taking (16) we can rewrite (15) as

$$
A_{i N}=\left|\beta_{1}^{\prime i N}\right\rangle\left\langle\alpha_{1}^{i N}|+\cdots+| \beta_{n_{a}^{i N}}^{i N}\right\rangle\left\langle\alpha_{n_{a}^{i N}}^{i N}\right|
$$

Obviously,

$$
\left[\left|\beta^{i N}\right\rangle\right]=[u]\left[\left|\beta^{i N}\right\rangle\right]
$$

Eq. (20) can be expressed explicitly as similar as Eq.(18). $\left\{\left|\beta_{1}^{i N}\right\rangle, \cdots,\left|\beta_{n_{a}^{i N}}^{i N}\right\rangle\right\}$ is a set of physical states, the mixture of these states, 


$$
\rho=\sum_{j=1}^{n_{a}^{i N}}\left|\beta_{j}^{i N}\right\rangle\left\langle\beta_{j}^{i N}\right|
$$

is a density matrix. (if $\operatorname{Tr} \rho \neq 1, \rho$ multiplied by a constant became a density matrix). The density matrix $\rho$ has always a set of eigenstates $\left\{\left|\beta_{1}^{\prime \prime i N}\right\rangle, \cdots,\left|\beta_{n_{a}^{i N}}^{\prime \prime i N}\right\rangle\right\}$ (unnormalized) such that

$$
\rho=\sum_{j=1}^{n_{a}^{i N}}\left|\beta_{j}^{\prime \prime} i N\right\rangle\left\langle\beta_{j}^{\prime \prime i N}\right|
$$

According to Wootters' criterion [20], the two sets of pure states, $\left\{\left|\beta_{1}^{\prime \prime i N}\right\rangle, \cdots,\left|\beta_{n_{a}^{i N}}^{\prime \prime i N}\right\rangle\right\}$ and $\left\{\left|\beta_{1}^{i N}\right\rangle, \cdots,\left|\beta_{n_{a}^{i N}}^{i N}\right\rangle\right\}$ fulfill

$$
\left[\left|\beta^{\prime \prime i N}\right\rangle\right]=[A]\left[\left|\beta^{i N}\right\rangle\right]
$$

where $[A]$ is a matrix the columns of which form a set of orthogonal bases. If the two sets have the same number of pure states, $[A]$ is a unitary matrix (since $\left\{\left|\beta_{1}^{i N}\right\rangle, \cdots,\left|\beta_{n_{a}^{i N}}^{i N}\right\rangle\right\}$ is a set of linearly independent vectors, $[A]$ in (23) is a unitary matrix). So we can always find a unitary matrix so that $\left\{\left|\beta_{1}^{\prime i N}\right\rangle, \cdots,\left|\beta_{n_{a}^{i N}}^{i N}\right\rangle\right\}$ in (20) is a set of orthogonal (possibly unnormalized) states. Namely, we can always express $A_{i N}$ as in (19) such that $\left\{\left|\beta_{1}^{i N}\right\rangle, \cdots,\left|\beta_{n_{a}^{i N}}^{i N}\right\rangle\right\}$ and $\left\{\left|\alpha_{1}^{i N}\right\rangle, \cdots,\left|\alpha_{n_{a}^{i N}}^{i N}\right\rangle\right\}$ is orthogonal vectors, respectively. Normalizing the states $\left\{\left|\beta_{1}^{\prime i N}\right\rangle, \cdots,\left|\beta_{n_{a}^{i N}}^{\prime i N}\right\rangle\right\}$ in (19), we get an expression of $A_{i N}$ as in (2). Similarly for $B_{i N}$ and $C_{i N}$. 


\section{REFERENCES}

[1] A. Einstein, B. Podoscky and N. Rosen, Phys. Rev 47, 777(1935)

[2] A. K. Ekert, Phys. Rev. Lett 67, 661(1991)

[3] C.H. Bennett, Phys. Rev. Lett 68, 3121(1992)

[4] C. H. Bennett, G. Brassard, C. Crepeau, R. Jozsa, A. Peres and W. K. Wootters. Phys. Rev. Lett 70, 1895(1993)

[5] A. Barenco, Contemp, Phys 37, 375(1996)

[6] C.H. Bennett, D.P. DiVincenzo, C.A. Fuchs, T.Mor, E.Rains, P.W. Shor, J.A. Smolin, and W.K. Wootters, Phys. Rev. A 59,1070 (1999)

[7] J. Walgate and L. Hardy, Phys. Rev. Lett 89, 147901(2002)

[8] M. Horodecki, A. Sen (De), U. Sen and R. Horodecki, quant-ph/0301106 (2003)

[9] J.Walgate, A.J.Short, L.Hardy and V.Vedral, Phys.Rev.Lett.85,4972 (2000).

[10] S.Ghosh, G.Kar, A.Roy, A.Sen and U.Sen, Phys.Rev.Lett.87, 277902 (2001); S. Ghosh, G.Kar, A.Roy, D.Sarkar, A.Sen(De) and U.Sen, quant-ph/0111136 (2001);

[11] S. Virmani, M.F. Sacchi, M.B. Plenio and D. Markham, Physics Letters A 288, 62-68 (2001);

[12] M. Horodecki, P. Horodecki, and R. Horodecki, Acta Physica Slovaca, 48, (1998) 141

[13] L.-M Duan and G.-C Guo, Phys. Rev. Lett 80, 4999(1998)

[14] Y.-X.Chen and D.Yang, Phys.Rev.A 64, 064303 (2001);

[15] P.-X Chen and C.-Z Li, quant-ph/0209048

[16] V.Vedral and M.B.Plenio, Phys.Rev.A 57, 1619 (1998)

[17] M. Horodecki, P. Horodecki and R. Horodecki, Phys. Rev. Lett 80, 5239(1998); N. Linden, S. Massar and S. Popescu, Phys. Rev. Lett 81, 3279(1998)

[18] J. Finkelstein, private communication.

[19] M. A. Nielsen and I. L. Chuang, Quantum computation and quantum information, p84-p93, Cambridge university Press.

[20] W. K.Wootters, Phys. Rev. Lett 80, 2245(1998); P.-X Chen et al, Phys. Rev. A 63, 052306(2001). 